\title{
Tracking Atria and Ventricles Simultaneously from Cardiac Short- and Long-Axis MR Images
}

\author{
J. Lötjönen ${ }^{1}$, D. Smutek ${ }^{1}$, S. Kivistö ${ }^{2}$, and K. Lauerma ${ }^{2}$ \\ 1 VTT Information Technology, P.O.B. 1206, FIN-33101 Tampere, Finland \\ Jyrki.Lotjonen@vtt.fi \\ 2 Department of Radiology, Helsinki University Central Hospital \\ P.O.B. 340, FIN-00029 HUS, Finland
}

\begin{abstract}
We propose a new approach for tracking the cardiac motion by using simultaneously short-axis and long-axis MR images in non-rigid registration. The fusion of two image orientations allows to track more precisely the basal and apical movement of the ventricles. In addition, the motion of the atria is tracked. Correlation coefficients were 0.94 at the systolic phase and 0.97 at the diastolic phase as the volumes of manually and automatically detected chambers were compared. The results were obtained using 7 subjects.
\end{abstract}

\section{Introduction}

Since cardiovascular disease is the most common cause of death in the Western countries, there is a strong need to diagnose and to quantify cardiac diseases early enough. The cardiac imaging techniques have improved considerably during the last years providing nowadays detailed anatomic and functional information on the heart. In addition, the automated analysis of the cardiac images has also been intensively developed [1].

Segmentation of cardiac images is a pre-requisite for obtaining many cardiac indices, such as left ventricular volume and ejection fraction. Several approaches have been proposed for the automated segmentation of the ventricles and/or myocardium from MR images [2 3,4]. Cine datasets contain images normally from 10-30 cardiac phases and produce therefore detailed information on the cardiac function during the cycle. Tagged MR images provide a golden standard for the cardiac motion analysis, as the tags visible in the images are tracked [5 6.7]. However, the analysis of cardiac function in clinical practice is still often based on standard MR images and visual inspection. Although segmentation tools developed for cardiac segmentation could basically be applied to each time instant separately, the techniques based on deformable models provide a natural framework for tracking the motion and changes in chamber volumes within the cine datasets. The tracking of the cardiac motion based on non-rigid registration of image volume has been demonstrated, for example, in [8,9].

To our knowledge the tracking of the heart has thus far concentrated only on ventricles and/or epicardium using short-axis images. Because the slice thickness is much higher than the pixel size in slice level, the tracking of the basal and apical regions of the ventricles using short-axis images is difficult. In practice, the ventricles are usually simply cutted by a plane at some basal level. In this paper, the non-rigid registration 
algorithm [9] was extended to track simultaneously short-axis (SA) and long-axis (LA) images. The LA images provide comprehensive information especially on tracking the movement of the basal and apical regions of the ventricles in the heart's long-axis direction. In addition, the atria were tracked during the non-rigid registration.

\section{Methods}

\subsection{Materials}

Seven healthy adults were studied. They were scanned using a 1.5 T Siemens Magnetom Vision imager (Siemens, Erlangen, Germany) at the Department of Radiology in Helsinki University Central Hospital (HUCH). During imaging sessions SA and LA cine images were acquired using a standard imaging protocol adopted for cardiac patients in HUCH: SA images contained ventricles from valve level until the level where the apex was still visible, and LA images contained atria and ventricles. In practice, a slight tilt compared to the standard LA view is used in HUCH in order to get the mitral and tricuspidal valves to the same image slice. The pixel size was $1.4 \times 1.4 \mathrm{~mm}$ for the SA and LA images. The slice thickness was $7 \mathrm{~mm}$ for the SA images and $7 \mathrm{~mm}$ for the LA images. The corresponding values for the slice separation were $15 \mathrm{~mm}$ and $10 \mathrm{~mm}$. The number of slices varied from 4 to 8 depending on the size of the heart. From 22 to 30 time points were taken for each section with a repetition time of $33 \mathrm{msec}$.

\subsection{Segmentation}

Atria, ventricles and epicardium were manually segmented from the images. A software tool designed for interactively making 3-D deformations was used. With it a triangulated surface model was registered manually and non-rigidly to edges in the SA and LA volumes. A segmentation result for one subject is shown in Fig. 1.

The SA and LA volumes were interpolated isotropic using a shape-based interpolation algorithm before segmentation [10]. Thereafter, the prior model was manually deformed to the SA volume. After finishing the deformation in the SA volume, the deformed model was transformed to the co-ordinate system of the corresponding LA volume and the matching was continued in the LA volume. The deformed model was transferred iteratively between the volumes until no deformations were needed. The procedure used is shown in Fig. 2

The rigid transformation, $\mathbf{f}$, used to transform the model from the SA volume to the LA volume ( $\mathbf{f}^{-1}$ from LA to SA) was extracted from image file headers which contained the orientation and the position of the slices relative to the patient's anatomy. In practice, eight points were chosen from the corners of the SA volume (cube) and their co-ordinates in the LA volume were defined based on the header information. Thereafter, a markerbased registration approach, i.e. the approach that minimized the distance between the corresponding points in the SA and LA volumes, was used to define translation and rotation.

Because all images were obtained at breath hold, we detected differences in the position of the heart between the breath holds. If the differences were not corrected, the transformation derived from the image headers would not not produce good results. In 


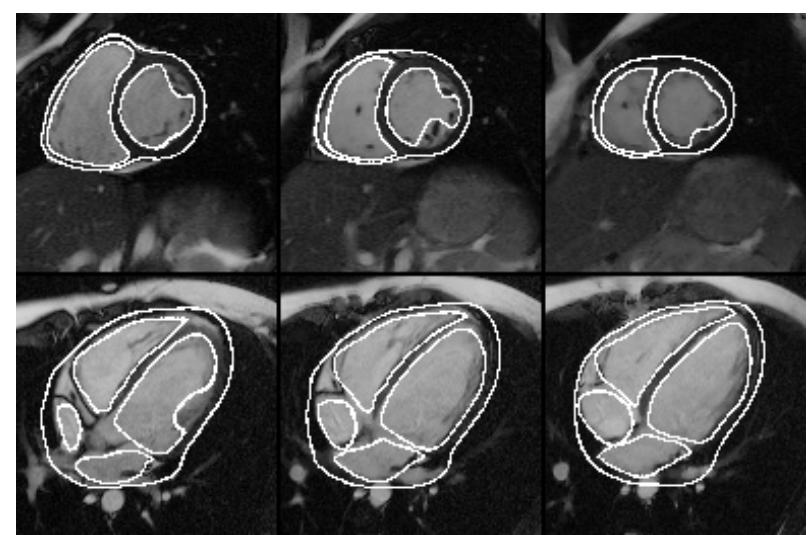

Fig. 1. A segmentation result for one subject shown on a few slices. A triangulated model has been superimposed on the SA volume (the top row) and on the LA volume (the bottom row). The rigid transformation $\mathbf{f}$ was used to transfer the model to the co-ordinate system of the LA volume.

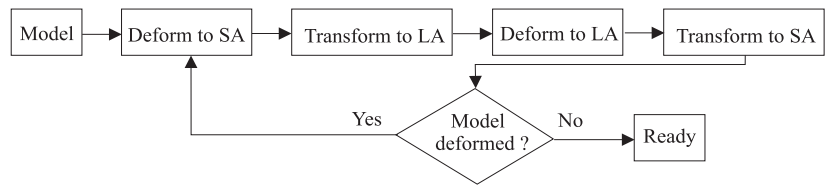

Fig. 2. Segmentation flow-chart.

this study, the differences were detected and corrected separately in each image volume. Image sets orthogonal to original slice orientation were generated and the slices were moved manually, if any abnormal discontinuities were detected in structures. The slice misalignments were corrected from five subjects.

\subsection{Non-rigid Registration}

In this paper, SA and LA volumes were registered non-rigidly simultaneously to different time instants. Formally, the source volumes $S_{1}(x, y, z)$ and $S_{2}(x, y, z)$ are registered to destination volumes $D_{1}(x, y, z)$ and $D_{2}(x, y, z)$, respectively. The objective of non-rigid registration is to find an optimal transformation $\mathbf{T}$ for aligning the source volumes to the destination volumes. The transformation is determined by maximizing a similarity measure between the volumes.

Similarity Measure. Normalized mutual information (NMI) was used as a similarity measure between the volumes $S_{i}$ and $D_{i}[11]$. The NMI is defined as:

$$
I\left(S_{i}, D_{i}\right)=\frac{H\left(S_{i}\right)+H\left(D_{i}\right)}{H\left(S_{i}, D_{i}\right)},
$$

where $H\left(S_{i}\right)$ and $H\left(D_{i}\right)$ are marginal entropies and $H\left(S_{i}, D_{i}\right)$ a joint entropy of the volumes $S_{i}$ and $D_{i}$. 


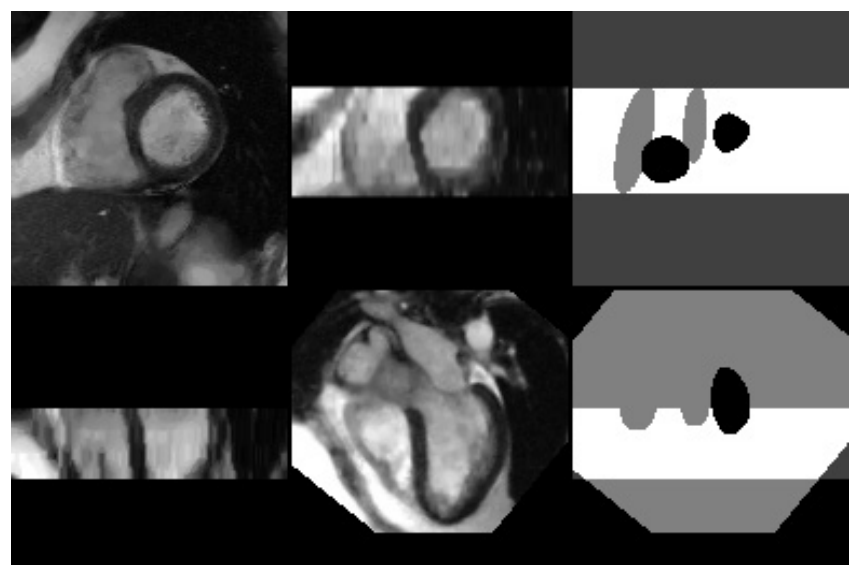

Fig. 3. The left and the middle columns show two orthogonal slices from the SA volume and the transformed LA volume, respectively. The right column shows the mask used in computing the NMI.

A combined similarity measure is defined as:

$$
I=I\left(S_{1}, D_{1}\right)+I\left(S_{2}, D_{2}\right)
$$

The computation of the measure is not straightforward during the non-rigid registration, because the orientation and the position of the SA and LA slices are not the same. If the non-rigid transformation $\mathbf{T}$ was defined for the SA volume, it should be rigidly registered using the rigid transformation, $\mathbf{f}$, in order to get the transformation in the LA co-ordinates. Making this transformation during the non-rigid registration would be very time consuming. Therefore, the LA volume is converted to the SA co-ordinates by the transformation $\mathbf{f}^{-1}$ before the non-rigid registration. The operation decreases the quality of the LA images but it was considered acceptable compared to decrease in the computation time.

After transforming the LA volume to the SA co-ordinates, all transformed LA voxels are not inside the SA volume. Therefore, empty (black) slices were added to the SA volume above the basal slice and below the apical slice in order to get all LA voxels inside the SA volume. Fig. 3 represents two orthogonal slices from the SA volume (the left column) and from the transformed LA volume (the center column).

The empty slices added should not be included in computing the NMI. Therefore, a mask was used to choose the voxels from the SA and LA volumes for the NMI computation, represented by the right column in Fig. 3 Both SA and LA voxels under the white color are used in computing NMI while only LA voxels are used for the light gray and only SA voxels for dark gray. The voxels under the black color are not encountered for the NMI. The reason for the round structures in the mask is explained in Section 2.4

The combined similarity measure including the mask and the transformed LA volume is defined as: 


$$
I=I_{M_{1}}\left(S_{1}, D_{1}\right)+I_{M_{2}}\left(S_{2}^{*}, D_{2}^{*}\right),
$$

where $M_{i}$ represents the mask for the volume $i$ and the superscript ' ${ }^{*}$ ' stands for the transformed volume, i.e. the volume one is assumed to be the SA volume and the volume two the LA volume.

Volumetric Deformation. The non-rigid registration of volume data was done by using the deformation sphere approach [9]. The approach is shortly summarized.

In the deformation sphere framework, the transformation is applied only to voxels or data points inside a sphere. The transformation vector $\mathbf{u}$ inside the sphere is defined as:

$$
\mathbf{u}=\frac{e^{-k \frac{\left(x-c_{x}\right)^{2}+\left(y-c_{y}\right)^{2}+\left(z-c_{z}\right)^{2}}{r^{2}}}-e^{-k}}{1.0-e^{-k}} \mathbf{U},
$$

where $k$ is a user-defined parameter (here 2.0), $\left(c_{x}, c_{y}, c_{z}\right)$ is the location of the sphere center, $r$ is the radius of the sphere (here from 30 voxels to 12 voxels) and $\mathbf{U}$ is the vector in the center of the sphere. During the deformation, the location and the size of the sphere is varied. The location of the sphere (the center of the sphere) is randomly located to the surfaces of atria, ventricles and epicardium. However, as the transformation is volumetric, the transformation is not only restricted to object boundaries. At each location of the sphere, the optimal transformation vector $\mathbf{U}$ is defined, i.e. the vector that maximize the similarity between the volumes. In practice, the vector $\mathbf{U}$ is defined as follows:

$$
\mathbf{U}=\arg \max _{I}\left\{\mathbf{p}+\mathbf{n}_{p}, \mathbf{p}, \mathbf{p}-\mathbf{n}_{p}\right\},
$$

where $I$ stands for the NMI, $\mathbf{p}$ is the location of the sphere's center and $\mathbf{n}_{p}$ is the normal vector of the surface (atrium, ventricle or epicardium) at location $\mathbf{p}$. A high number of spheres (a few thousands) are sequentially applied to the data during the deformation. The coarse-to-fine approach was used: the size of the sphere is gradually decreased during the iteration.

\subsection{Volume Tracking}

The volume tracking was done as in [89]: the volume of time instant $t$ was matched to the volume of time instant $t+1$. The transformation $\mathbf{T}_{0, t}$ from the time instant 0 to the time instant $t$ can be computed from

$$
\mathbf{T}_{0, t}=\sum_{i=0}^{t} \mathbf{T}_{i, i+1} .
$$

As the tracking protocol was developed and tested, the tracking of the valve level was problematic. Fig. 4 a shows the function of the mitral valve (indicated by an arrow) during three consecutive time phases at systolic phase. The detection of the border between the ventricles and the atria is clear when the mitral and tricuspid valves are closed. However, when the valves open, the tracking algorithm should not follow the valves as happens if 


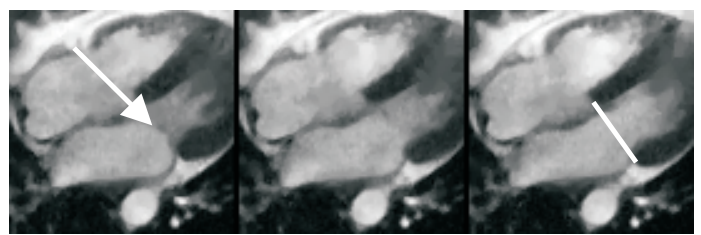

(a)

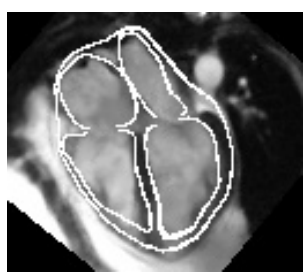

(b)

Fig. 4. a) Three consecutive time instances during the systolic phase. The mitral valve is indicated by an arrow and the desired border between the left atrium and ventricle while the valve is open, by a line. b) The surfaces used in non-rigid registration.

the deformation is not regulated. It was decided that the border should be on the straight line between the most basal parts of the myocardium (indicated by a line in Fig. 4 a).

Two solutions were proposed to cope with the problem of opening valves: modification of the mask and modification of the surfaces of the objects of interest.

Modification of the Mask. Because the opening valves disturb the tracking algorithm, the valves were masked out from the volume data. In practice, the user placed manually ellipsoid shaped surfaces near both valves at the time phase $t=0$ (the most diastolic phase) and deformed it manually to contain the valves using the same software tool as described in Section 2.2 Thereafter, the volumes inside the surfaces were deleted from the mask (black round regions in Fig. (3). Because the transformation $\mathbf{T}$, defined to the SA and LA volumes, was applied to the surfaces during the deformation, the interaction was needed only for the first time phase. As mentioned above, the valves disturb the deformation only while opening. Therefore, the masking of the valves was used only after the systolic time phase. In practice, in all studied cased the masking was started from the time phase 10 when the valves were still closed.

Because the resolution of the SA images in long-axis direction (orthogonal to the slice) is fairly poor compared to the LA images, the masking out of the basal regions of the myocardium from the SA images was also applied. The protocol described in the previous paragraph was used, i.e. the user manually placed ellipsoid shaped surfaces surrounding the basal parts of the myocardium (light gray round regions in Fig. 3). The masking was started from the time phase 0 .

Modification of the Surfaces of the Objects of Interest. As mentioned in the previous section, the deformation spheres were randomly placed only to the surfaces of atria, ventricles and epicardium. Because the tracking of the valves was not desired, parts of the surfaces near the valves were deleted. In practice, the triangulated surfaces were used, and triangles close to valves were removed. Fig. 4,b shows the surfaces superimposed on one slice after deleting the triangles from the valve region. Since the transformation is volumetric, the valve region is also transformed but based on the transformations applied in surrounding regions, i.e. the basal regions of the myocardium.

\section{Results}

The comparison of volumes between manual and automatic volume tracking is shown in Fig. 5. The results are shown both for the systolic and diastolic phases. The diastolic 

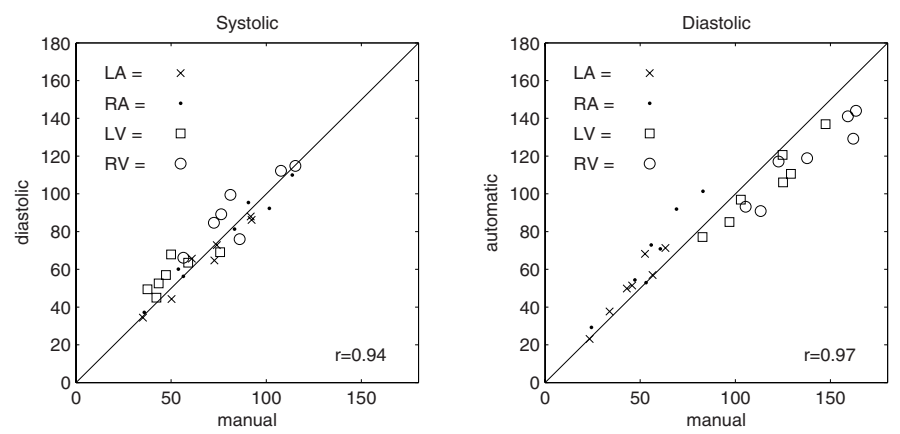

Fig. 5. The comparison of volumes produced by manual and automatic segmentation at the systolic (left) and diastolic (right) time phases. The diagonal line demonstrates the perfect agreement between manual and automatic results.

phase contains cumulative error of all deformations from the diastolic phase $(t=0)$ to diastolic phase ( $t=22-30$ ). The cumulative error is, however, built up from signed contributions meaning that if, for example, no elasticity is allowed to the model, the error could be zero at the diastolic phase although the error was large in the systolic phase. The correlation coefficients were 0.94 and 0.97 at the systolic and diastolic phases, respectively. The computation time for registering 24 time phases was about two hours ( 5 minutes/registration) using a standard $2.0 \mathrm{GHz}$ Pentium Workstation.

\section{Discussion}

In this paper, a new approach was proposed to volume tracking of atria, ventricles and epicardium. The approach combined information both from standard SA and LA cardiac images. This made possible the tracking of the atria as well as the basal and apical regions of the ventricles.

A high correlation $(r=0.94-0.97)$ between manual and automatic tracking was achieved. At the diastolic phase, the volumes of ventricles were underestimated and volumes of atria overestimated in each studied case. For the systolic phase this effect was not found. One reason for the bias at the diastolic phase was the problem of valve tracking, i.e. the basal level did not return always correctly to the original level (level at $t=0$ ). The approaches proposed in Section 2.4 improved the accuracy considerably. In addition to the improvements in the algorithm, the data acquisition could obviously be optimized. However, the use of standard datasets is a srength as the clinical use of the method is considered.

Future work will concentrate on automating the manual steps of the protocol. In the current version, the first time phase was manually segmented and the modifications to the mask on the basal level were made manually. The misalignments of slices due to patient movement were also corrected manually.

Acknowledgements Research was supported by the National Technology Agency, Finland. 


\section{References}

1. A.F. Frangi, W.J. Niessen and M.A. Viergever. Three-Dimensional Modeling for Functional Analysis of Cardiac Images: A review. IEEE Trans. Med. Imag., 20(1): 2-25, 2001.

2. L.H. Staib and J.S. Duncan. Model-based deformable surface finding for medical images. IEEE Trans. Med. Imag., 15(5): 720-731, 1996.

3. M-P. Jolly. Combining Edge, Region, and Shape Information to Segment the Left Ventricle in Cardiac MR Images. Lecture Notes in Computer Science 2208: Medical Image Computing and Computer-Assisted Intervention - MICCAI 2001, Eds. W.J. Niessen and M.A. Viergever, pp. 482-490, 2001.

4. S.C. Mitchell, J.G. Bosch, B.P.F. Lelieveldt, R.J. van der Geest, J.H.C. Reiber and M. Sonka. 3-D Active Appearance Models: Segmentation of Cardiac MR and Ultrasound Images. IEEE Trans. Med. Imag., 21(9): 1167-1178, 2002.

5. S. Kumar and D. Goldgof. Automatic tracking of SPAMM grid and the estimation of deformation parameters from cardiac MR images. IEEE Trans. Med. Imag., 13(1): 122-132, 1994.

6. Y. Chen and A.A. Amini. A MAP Framework for Tag Line Detection in SPAMM Data Using Markov Random Fiels on the B-Spline Solid. IEEE Trans. Med. Imag., 21(9): 1110-1122, 2002 .

7. A. Montillo, D. Metaxas and L. Axel. Automated Segmentation of the Left and Right Ventricles in 4D Cardiac SPAMM Images. Lecture Notes in Computer Science 2488: Medical Image Computing and Computer-Assisted Intervention - MICCAI 2002, Eds. D. Dohi and R. Kikinis, pp. 620-633, 2002.

8. M. Lorenzo-Valdés, G.I. Sanchez-Ortiz, R. Mohiaddin and D. Rueckert. Atlas-Based Segmentation and Tracking of 3D Cardiac MR Images Using Non-rigid Registration. Lecture Notes in Computer Science 2488: Medical Image Computing and Computer-Assisted Intervention - MICCAI 2002, Eds. D. Dohi and R. Kikinis, pp. 642-650, 2002.

9. J. Lötjönen. Segmentation of MR Images using Deformable Models: Application to Cardiac Images. International Journal of Bioelectromagnetism, 3(2), 2001, http://ee.tut.fi.rgi/ijbem.

10. G.J. Grevera and J.K. Udupa. Shape-Based Interpolation of Multidimensional Grey-Level Images. IEEE Trans. Med. Imag., 15(6): 881-892, 1996.

11. C. Studholme, D.L.G. Hill and D.J. Hawkes. Automated three-dimensional registration of magnetic resonance and positron emission tomography brain images by multireolution optimization of voxel similarity measures. Medical Physics, 24(1): 71-86, 1997. 